\title{
Recepción de series televisivas y formación de esquemas cognoscitivos asociados a sus personajes: un estudio con niños y adolescentes costarricenses*
}

\author{
Reception of Television Series and Formation \\ of Cognitive Schemas Associated to their Characters: \\ A Study of Costa Rican Children and Adolescents
}

Recibido: febrero 23 de 2011 | Revisado: octubre 11 de 2011 | Aceptado: diciembre 3 de 2011

\author{
ROLANDO PÉREZ SÁNCHEZ** \\ DAVID TORRES FERNÁNDEZ \\ Universidad de Costa Rica, Costa Rica
}

RES UMEN

El estudio indaga la asociación entre el visionado televisivo, específicamente la intensidad de uso de la televisión y la valoración de series de televisión, en la formación de esquemas de personas. Compara la organización categorial ante una serie de televisión en un grupo de niños, niñas y adolescentes. Se estudiaron variables cognoscitivas y psicomediáticas, como variables intervinientes. Los resultados muestran diferencias entre los grupos de edad con respecto a la accesibilidad y variabilidad del contenido de los esquemas y la participación diferencial de las variables cognoscitivas y psicomediáticas. Se discuten sus implicaciones para el estudio de la comprensión del mensaje televisivo, específicamente de las series televisivas, y para el desarrollo de las competencias para la recepción televisiva.

Palabras clave autores

Recepción de series de televisión, cognición social, esquemas de personas, psicología de los medios de comunicación.

Palabras clave descriptores

Medios de comunicación, niños, adolescentes.

\section{A B S T R A C T}

The study inquires the association between television viewing, specifically television use intensity and TV series evaluation on the formation of person schemata. It compares categorical organization among a group of children and adolescents facing a TV series. As intervening variables are studied cognitive and media psychological variables. Results show age differences concerning accessibility and variability of schemata content and differential participation of cognitive and media psychological variables. Implications for the study of the comprehension of TV series and for the development of competences for TV reception are discussed.

Key words authors

TV Series Reception, Social Cognition, Person Schemata, Media Psychology.

Key words plus

Mass Media, Children, Adolescents. 


\section{Introducción}

El presente estudio se enmarca dentro del interés general por estudiar la formación de esquemas de personas en la recepción de series de televisión en niños, niñas y adolescentes. Específicamente, pretende indagar la relación entre la intensidad de uso televisivo, por un lado, la valoración de la serie visionada, por otro, y la complejidad de los esquemas de personas asociados a personajes de series de televisión, considerando variables propias de la infraestructura cognoscitiva como la memoria declarativa y las competencias narrativas, así como variables socioemocionales implicadas en la recepción de series de televisión.

La investigación retoma los modelos sociocognoscitivos de la recepción mediática (Collins, 1981; Shrum, 2006; Wicks, 2006). En ellos, se considera necesario tomar en cuenta las relaciones entre los factores emocionales, la infraestructura mnémica y los esquemas cognoscitivos, e implica considerar la asociación entre los marcos interpretativos individuales propios del receptor y los mediáticos, entendidos como redes compartidas para la comprensión del mensaje mediático.

En este estudio interesa, específicamente, acercarse a las representaciones de personajes mediáticos de ficción, considerando como eje conceptual el estudio de los esquemas de personas. Se entenderá aquí por esquemas de personas, 'las estructuras cognoscitivas de categorización social que posibilitan la codificación, almacenamiento y la realización de inferencias sobre tipos sociales' (Fiske $\&$ Taylor, 2008). La categorización social permite clasificar, describir y explicar el comportamiento de otras personas de conformidad a características físicas, de personalidad, relacionales, morales o por su pertenencia a agrupaciones socialmente construidas, sean estas ocupacionales, etarias, por género, etnoculturales o por posición social, entre otros. Además, se estructura atendiendo a procesos conceptualmente conducidos, es decir, mediante el procesamiento de la información conforme a esquemas previamente estructurados. La accesibilidad a estos esquemas está supeditada a su disponibilidad en la memoria de trabajo y sus posibilidades de recuperación por medio de la asociación entre la información nueva y la precedente.

Ahora bien, la formación de la representación de los personajes televisivos supone recurrir a procesos automáticos, ya sea mediante la atención a ejemplares (es decir, personas similares ofrecidas por los encuadres mediáticos inmediatos, la experiencia previa o por la experiencia extramediática), así como mediante la activación de representaciones prototípicas o modelos estereotípicos construidos culturalmente. El procesamiento sociocognoscitivo propio del visionado de series televisivas supone que los ofrecimientos de la serie ejercen un efecto de priming, y es precisamente mediante el recurso a ejemplares o prototipos como se va a configurar el esquema de persona específico, sus rasgos característicos y su complejidad (Busselle, 2001; Shrum, 2006).

El encuadre narrativo de las series se caracteriza por construir el perfil de los personajes de forma estereotípica, esto facilita la identificación con los personajes y la predicción de su comportamiento y del conjunto de la serie, por parte de la persona espectadora. Al interior de los encuadres narrativos, esta construcción estereotípica de los caracteres se asocia con personas o roles de la vida real. Esta cualidad favorece no solo la identificación con el personaje, sino su valoración y la de la serie en general (Pérez, 2003a).

Estudios previos que indaguen la estructuración y contenido de los esquemas, particularmente en su complejidad, son escasos. En la década del 80 al 90 del siglo pasado, se publicaron investigaciones relevantes relacionadas con esta temática y enmarcadas dentro del estudio de la asociación entre la formación de estereotipos y el visionado televisivo, haciendo énfasis en los esquemas de rol, particularmente ocupacionales (Calvert \& Huston, 1987; Wright, Huston, Fitch, Wroblewski \& Piemyat, 1997; Wright et al., 1995; Wroblewski \& Huston, 1987), y la identidad social, y considerando estereotipos etarios, hacia minorías étnicas y hacia las mujeres (Ford, 1997; Harwood, 1999; Mastro, 2003; Mastro \& Kopacz, 2006). Mientras que los trabajos que evalúan la asociación entre la intensidad de uso televisivo y la evaluación cognoscitiva implicada en 
la definición de los esquemas, se puede asociar al estudio del efecto de cultivo y los heurísticos cognitivos implicados (Shrum, 2006). En lo que respecta a la valoración de la serie, esta se puede asociar a la línea de estudios enmarcados dentro del enfoque de los usos y gratificaciones, que centra su atención en los aspectos motivacionales y emocionales de la interacción entre la persona receptora y el medio (Pérez, 2003a, 2003b; Rubin, 2002).

El presente estudio es un aporte a la comprensión de la complejidad del esquema y al estudio de los mecanismos cognitivos y aquellos propios de la interacción mediática que puedan estar asociados a su formación. La complejidad del esquema se refiere aquí a la cantidad y diversidad de rasgos que caracteriza la descripción de un personaje. Las variaciones en la complejidad pueden estar asociadas a determinantes propios del procesamiento cognoscitivo implicado en la recepción y apropiación de series televisivas, como son la memoria declarativa y las competencias narrativas que definen los contenidos almacenados así como la complejidad de su recuperación.

Igualmente, las dimensiones asociadas a la interacción entre el contenido mediático y el receptor, que se seguirán llamando dimensiones psicomediáticas, pueden definir la complejidad del esquema. Específicamente, la identificación parasocial con los personajes de las series (Rubin \& Perse, 1987), la motivación por el uso televisivo y la implicación con su contenido (Ward \& Rivadeneyra, 1999), son variables que definen el compromiso emocional y disposición cognoscitiva para la formación de esquemas de personajes en una serie de televisión.

Los resultados que a continuación se exponen presentan como variables independientes la intensidad del uso televisivo y la valoración de la serie sobre la complejidad del esquema. Se consideró relevante evaluar la intensidad de uso, atendiendo a los resultados de la teoría del cultivo (Shanahan \& Morgan, 2003), que señala el papel del visionado televisivo intenso y prolongado sobre las visiones de mundo, y en este caso particular sobre la complejidad del esquema. La valoración de la serie es un elemento de evaluación socioemocional que ha sido considerado importante tanto por el enfoque de usos y gratificaciones como por la investigación sobre la interacción parasocial con los contenidos mediáticos y podría estar definiendo la conformación del esquema (Pérez, 2003b; Rubin, 2002). El resto de variables consideradas en los párrafos anteriores, es decir, las variables cognitivas (memoria declarativa y complejidad narrativa) y las variables psicomediáticas (identificación parasocial, motivación e implicación hacia el visionado televisivo) son consideradas como variables control en la relación entre la complejidad del esquema y la intensidad de visionado, y la valoración de la serie.

Es importante señalar que, en Costa Rica, la oferta televisiva de programas de ficción está compuesta en su gran mayoría por programas extranjeros, provenientes de Estados Unidos y Latinoamérica. Dentro de estos programas, se encuentran las series de televisión el formato mayormente consumido por niños y jóvenes. La producción nacional de series es realizada por productoras privadas, presentada en canales privados y dirigida a público adulto o a formatos familiares (Pérez, 2003a).

En resumen, el problema de investigación que orienta el presente estudio se pregunta sobre la relación entre la intensidad de visionado televisivo y la valoración de las series de televisión sobre la formación de esquemas cognitivos de personajes televisivos en niños y adolescentes costarricenses. Asimismo, resulta relevante determinar el papel de funciones básicas en el procesamiento cognitivo como puede ser la memoria declarativa y las competencias narrativas, así como factores emocionales como la motivación de uso, la identificación parasocial o la implicación, en la recepción de series.

Las hipótesis del estudio son las siguientes:

$\mathrm{H}_{1}$ : Los adolescentes presentarán esquemas de personajes más complejos que los niños.

$\mathrm{H}_{2}$ : Se presentan diferencias entre niños y adolescentes en la asociación entre la valoración de la serie y la complejidad del esquema, de modo que para los niños resulta más relevante esta valoración que para los adolescentes.

$\mathrm{H}_{3}$ : Tanto para niños como para los adolescentes la intensidad de uso televisivo va a definir la complejidad del esquema, de tal manera que a mayor intensidad de uso mayor la complejidad. 
$\mathrm{H}_{4}$ : Las memoria declarativa y las competencias narrativas van a intervenir en la complejidad del esquema, con dependencia de la edad de los receptores, encontrándose un efecto positivo en los adolescentes, no así en los niños.

$\mathrm{H}_{5}$ : Las variables psicomediáticas (motivación, implicación e identidad parasocial van a intervenir en la complejidad del esquema, con dependencia de la edad, identificándose un efecto positivo en los niños, no así en los adolescentes.

\section{Método}

\section{Participantes}

El estudio se efectuó con un total de 125 participantes, 62 estudiantes de instituciones de enseñanza primaria, con un promedio de edad de 11.03 años $(D E=1.73), 26$ hombres y 36 mujeres, y 63 personas de instituciones de enseñanza secundaria con un promedio de edad de 14.5 años $(D E=1.34), 29$ hombres y 34 mujeres. Se recurrió a un muestreo de tipo intencional.

Los niños, niñas y adolescentes fueron seleccionados, mediante consulta a las personas encargadas del menor. Los padres, madres o encargados firmaron un consentimiento informado. Los adolescentes también firmaron el protocolo de consentimiento informado.

\section{Instrumentos}

Se elaboró un cuestionario autoaplicado, para uso tanto en niños como adolescentes que comprende las siguientes dimensiones: intensidad de uso televisivo, valoración de la serie, implicación general con el visionado televisivo, motivación hacia el visionado televisivo, identificación parasocial y variables sociodemográficas (edad y sexo).

Intensidad de visionado televisivo: se indagó mediante el número de horas por semana de consumo televisivo, para lo cual los participantes debían de marcar el número de horas de visionado diarias.

Valoración de la serie: se refiere a la evaluación positiva o negativa que hace el receptor de este género televisivo. Se exploró a partir de la califica- ción que hicieron los participantes en una escala de 0 a 10.

Implicación: se refiere al grado de compromiso emocional con la serie, expresado en la formación de rutinas alrededor de su visionado y en la incorporación de los contenidos de la serie dentro de las reflexiones y conversaciones cotidianas. Para el estudio de la implicación hacia el uso de la televisión se recurrió a la Escala de Visionado Activo (Ward \& Rivadeneyra, 1999), originalmente compuesta por 24 ítems, luego de la prueba piloto y los análisis psicométricos, se trabajó con una escala de 11 ítems. Los participantes respondieron a cada reactivo en una escala Likert de 4 puntos que van de 1 (totalmente en desacuerdo) a 4 (totalmente de acuerdo), con un alfa de Cronbach fue de 0.79. Ejemplos de los reactivos son: "Hay ciertos programas de televisión que nunca me pierdo", "Estoy viendo el reloj para no perderme mis programas de televisión favoritos", "A menudo pienso en cosas que vi en la televisión uno o dos días después".

Motivación de visionado: se entiende como las razones o impulsores que orientan el visionado de series de televisión. Se asocia con la adquisición de conocimiento sobre el mundo y el autocercioramiento. Se recurrió a las Escalas de Motivos de Visionado de Ward y Rivadeneyra (1999), compuesta en su versión original por 22 ítems. Luego de la prueba piloto y de los análisis psicométricos, se decidió emplear una versión de 7 ítems. Los participantes respondieron a cada reactivo en una escala Likert de 4 puntos que van de 1 (totalmente en desacuerdo) a 4 (totalmente de acuerdo). El alfa de Cronbach de los reactivos fue de 0.78. Algunos ejemplos de los ítems son: "Aprendo sobre mí mismo", "Aprendo sobre lo que puede pasarme en el futuro", "Me ayuda a entender el mundo".

Identificación parasocial: se refiere a la identificación que establece un receptor con un personaje televisivo -caracterizado por una sensación de cercanía y afinidad- similar a la que acontece en las relaciones cara a cara. Para estudiar la identificación parasocial, se recurrió a la escala del mismo nombre empleada por Rubin y Perse (1987). A partir de la prueba piloto y los análisis psicométricos se empleó una versión de 7 items 
(la escala original consta de 10). Los participantes respondieron a cada reactivo en una escala Likert de 4 puntos que van de 1 (totalmente en desacuerdo) a 4 (totalmente de acuerdo). El alfa de Cronbach de los reactivos fue de 0.84 . Ejemplos de los ítems son: "Espero ver a mi personaje de televisión favorito en el episodio de esta semana", "Me gustaría conocer en persona a mi personaje favorito de televisión", "Mi personaje de televisión favorito me hace sentirme tan cómodo como cuando estoy con mis amigos".

Dado que era la primera vez que en el país se hacía uso de estas tres escalas, se procedió a su adaptación. Se hizo una traducción que fue revisada por expertos. Esta versión fue evaluada mediante la Entrevista Cognoscitiva para la Elaboración de Instrumentos (Willis, 2004), para lo cual se realizaron dos entrevistas grupales con niños y niñas y dos con adolescentes.

\section{Procedimiento}

La primera fase del estudio fue la escogencia de una serie de televisión que funcionara de estímulo, con la condición de que no fuera un programa de dibujos animados, sino que presentara personajes reales. Como criterio para la selección de la serie, se consideraron los ratings para los grupos de edad en estudio. Asimismo, se efectuó un sondeo para asegurarse de que la serie fuese de alto consumo entre la población en estudio. Con ello se pretendía controlar que la accesibilidad de los esquemas asociados a los personajes no se viese afectado por la falta de exposición previa a la serie seleccionada. Se encontró que los dos grupos veían series propias de su edad, por lo que fue necesario escoger una serie para el grupo de niños y otra para adolescentes.

Para el grupo de niños se seleccionó Zack y Cody, que es una sitcom (comedia de situación) infantil, que relata la vida de dos niños gemelos que viven en un hotel junto con su madre que es la cantante de dicho hotel. Tiene una duración aproximada de 30 minutos. Para el grupo de los adolescentes se escogió la serie policiaca CSI, la cual presenta a un grupo de criminalistas y su trabajo. En cada capítulo se escenifica la manera en la que se resuelven dos casos. La duración de cada episodio es de 45 minutos.

En primer término, los participantes llenaron el cuestionario previamente descrito. Posteriormente, a cada participante se le citó en una de las oficinas de su institución escolar. Allí procedían a ver un capítulo de la serie, seleccionado para su grupo de edad. Una vez finalizado el visionado del episodio, se realizó una entrevista semiestructurada que iniciaba con una narración espontánea de la serie en general y del capítulo en particular. Seguidamente, se les solicitaba a las personas que nombraran todos los personajes que recordaran en el episodio. Luego, se le pedía que realizaran una descripción espontánea de cada uno de los personajes nombrados. Por último, se procedía con una descripción dirigida que comprendía rasgos físicos, psicológicos, sociales, morales y roles sociales de los personajes recordados.

La información obtenida en esta fase se procesó mediante análisis de contenido (Krippendorf \& Rock, 2008), recurriendo al paquete informático Atlas.ti. La información se codificó atendiendo a las siguientes dimensiones: 1) Número de palabras, específicamente, sustantivos, adjetivos, verbos y adverbios, empleados en la narración espontánea acerca del capítulo de la serie observado. Esto se tomó como un criterio para evaluar la competencia narrativa. 2) Número de personajes recordados, que se empleó como criterio para evaluar la memoria declarativa. 3) La accesibilidad de los personajes (orden en el que se nombraron los personajes). 4) Número de rasgos enunciados por personaje. 5) Número de rasgos físicos (descripción física de los personajes, por ejemplo, color de pelo, estatura, contextura física, edad), psicológicos (características o disposiciones intrínsecas al personaje, por ejemplo, tímido, meticuloso, activo), sociales (formas de interactuar con otras personas, por ejemplo, mantienen buenas o malas relaciones con los otros personajes), morales (evaluaciones de las acciones de los personajes como buenas o malas) y roles sociales (funciones sociales de los personajes, por ejemplo, juez, testigo). 6) La sumatoria de los tipos de rasgos, se tomó como indicador de complejidad del esquema. La codificación fue realizada por un equipo de 
cuatro asistentes de investigación, dirigidos por el investigador principal, quienes fueron entrenados en la técnica y en el sistema de codificación. Con el propósito de garantizar la consistencia interna de la codificación, durante el proceso se llevaron a cabo reuniones grupales periódicas, con el objetivo de revisar lo codificado, unificar las interpretaciones de los criterios y realizar correcciones.

Los análisis se realizaron considerando los dos personajes más accesibles en las respectivas series. Esta decisión fue tomada debido a la estructura narrativa de las series televisivas, que supone la interacción entre dos o más personajes que se mantienen constantes a lo largo del tiempo, resultando precisamente dos el número de personajes mínimo a considerar. Para la serie CSI, los perso- najes más accesibles fueron Katherine y Grisson, en ese orden, y para Zack y Cody, los personajes que dan nombre a la serie. Es importante señalar que estos son los personajes principales en las respectivas series y los que se mantienen de episodio en episodio.

\section{Resultados}

\section{Correlaciones entre las variables en estudio}

En la muestra de niños no se encontró ninguna asociación entre la memoria y la complejidad de la narración y el resto de variables estudiadas, ni entre sí (Tabla 1).

TABLA 1

Media, desviación típica y correlaciones entre las variables en estudio para el grupo de niños

\begin{tabular}{lcccccccccc}
\hline & $\mathrm{M}$ & $\mathrm{DE}$ & $\mathbf{2}$ & $\mathbf{3}$ & $\mathbf{4}$ & $\mathbf{5}$ & $\mathbf{6}$ & $\mathbf{7}$ & $\mathbf{8}$ & 9 \\
\hline 1- Memoria & 47.31 & 5.62 & 0.21 & 0.00 & 0.08 & -0.05 & 0.09 & 0.08 & -0.06 & 0.04 \\
2- Complejidad narración & 95.37 & 143.06 & & 0.06 & 0.02 & -0.00 & 0.24 & 0.08 & 0.01 & 0.01 \\
3- Intensidad de uso de la TV & 36.89 & 18.71 & & & 0.20 & $0.34^{* *}$ & $0.31^{*}$ & $0.34^{* * *}$ & 0.00 & 0.25 \\
4- Motivación & 17.48 & 4.86 & & & & $0.49^{* *}$ & $0.49^{* *}$ & $0.28^{*}$ & 0.08 & $0.38^{* *}$ \\
5- Interacción parasocial & 19.90 & 5.6 & & & & & $0.57^{* *}$ & $0.34^{* *}$ & -0.09 & 0.20 \\
6- Implicación & 29.65 & 7.4 & & & & & & $0.44^{* * *}$ & -0.05 & $0.32^{*}$ \\
7- Valoración de la serie & 8.98 & 1.9 & & & & & & & 0.06 & 0.23 \\
8- Complejidad del esquema Zack (1) & 0.34 & 0.81 & & & & & & & & 0.20 \\
9- Complejidad del esquema Cody (2) & 1.73 & 0.79 & & & & & & & & \\
\hline
\end{tabular}

$* p<0.05 ; * p<0.01$.

Fuente: elaboración propia.

TABLA 2

Media, desviación típica y correlaciones entre las variables en estudio para el grupo de adolescentes

\begin{tabular}{lcccccccccc}
\hline & $\mathrm{M}$ & $\mathrm{DE}$ & 2 & 3 & 4 & 5 & 6 & 7 & 8 & 9 \\
\hline 1- Memoria & 115.73 & 23.46 & 0.21 & 0.01 & -0.23 & -0.10 & -0.00 & -0.05 & $0.41^{* *}$ & 0.15 \\
2- Complejidad narración & 92.65 & 62.61 & & -0.07 & -0.03 & 0.14 & 0.08 & 0.17 & $0.31^{* *}$ & $0.39^{* *}$ \\
3- Intensidad de uso de la TV & 35.37 & 14.75 & & & -0.15 & -0.07 & $0.31^{*}$ & 0.26 & -0.11 & -0.12 \\
4- Motivación & 15.4 & 4.44 & & & & $0.39^{* *}$ & $0.31^{*}$ & 0.06 & -0.06 & 0.11 \\
5- Interacción parasocial & 17.07 & 5.06 & & & & & $0.44^{* * *}$ & $0.30^{*}$ & -0.08 & 0.12 \\
6- Implicación & 28.1 & 5.86 & & & & & & 0.06 & -0.01 & 0.09 \\
7- Valoración de la serie & 7.91 & 2.65 & & & & & & & -0.02 & 0.03 \\
8- Complejidad del esquema grissom (1) & 1.57 & 0.95 & & & & & & & & $0.28^{*}$ \\
9- Complejidad del esquema Katherine (2) & 1.44 & 0.8 & & & & & & & & \\
\hline
\end{tabular}

$* p<0.05 ; * *<<0.01$.

Fuente: elaboración propia. 
La intensidad de visionado televisivo se asocia positivamente con dos de las variables psicomediáticas, a saber identificación parasocial y la implicación ( $r s=0.34,0.31$ ps $<0.01,0.05)$. Así, una alta intensidad televisiva parece asociarse tanto con una alta identificación parasocial como con una alta implicación hacia el uso televisivo. Igualmente, la intensidad de uso se asocia positivamente con la calificación de la serie $(r=0.34$, $p<0.01)$, de forma tal que altos puntajes en la intensidad de visionado se relacionan con una alta calificación de la serie.

Las variables psicomediáticas presentan una asociación positiva entre sí, resultando que una alta motivación de uso podría asociarse con una alta identificación parasocial y una alta implicación con el medio ( $r s=0.49,0.57, p<0.01$ ). Asimismo, la motivación y la implicación se relacionan positivamente con la complejidad del esquema del personaje Cody $(r=0.39,0.31, p<0.01,0.05)$, de modo que una alta motivación e implicación con el visionado se puede asociar con un esquema más complejo del personaje.

La calificación de la serie se asocia positivamente con todas las variables psicomediáticas, es decir, motivación, identificación parasocial y la implicación $(r s=0.28,0.34,0.44$, ps $<0.05,0.01,0.01$, respectivamente). De esta manera, altos puntajes en la calificación de la serie se asocian a altos puntajes en la motivación y la implicación respecto al visionado televisivo, además se relaciona con altos puntajes con respecto a la identificación parasocial con los personajes de televisión.

En el caso de los adolescentes (Tabla 2), la memoria no se relaciona con las variables psicomediáticas, únicamente con la complejidad del esquema del personaje 1 , con el cual se presenta una asociación positiva $(r=0.41, p<0.05)$, de modo que un mayor puntaje en la memoria declarativa parece asociarse una complejidad del esquema alta. De manera similar, la complejidad de la narración tampoco se asoció con las variables psicomediáticas estudiadas, pero sí se relacionó positivamente con la complejidad del esquema de ambos personajes estudiados ( $r s=0.31,0.39,=p s<0.05,0.01$ ), lo que indica que una mayor competencia narrativa parece asociarse con una mayor complejidad de los esquemas.

En cuanto a la intensidad de uso televisivo, esta variable se asoció positivamente con la implicación hacia el visionado televisivo $(r=0.31, p<0.05)$, de modo que a mayor el visionado mayor es la implicación.

Las variables que hemos llamado psicomediáticas, motivación, identificación parasocial e implicación, parecen asociarse entre sí de forma positiva $(r s=0.39,0.31,0.44$ ps $<0.01,0.05,0.001)$, de forma tal que, por ejemplo altos puntajes de motivación hacia el visionado televisivo se asocian con una alta identificación parasocial y un alto puntaje en implicación con el medio. Con otras variables no se presentan asociaciones.

Finalmente, en cuanto a la calificación de la serie, esta solo presenta una asociación positiva con la identificación parasocial $(r=0.30, p<0.05)$, de modo que una calificación alta se relaciona con una identificación parasocial también alta.

\section{Diferencias de medias entre los grupos en las variables en estudio}

En cuanto a la complejidad de los esquemas se encontraron diferencias en el personaje 1, resultando que los adolescentes presentan mayor complejidad que los niños $(t(123)=-7.548, p<0.001$, Niños: $\mathrm{M}=0.34, \mathrm{DE}=0.81$. Adolescentes: $\mathrm{M}=1.84$, $D E=1.35)$, como se muestra en la Tabla 3.

En cuanto a las variables cognoscitivas se encontraron diferencias significativas con la memoria. Se encontró que los adolescentes presentan mayores puntajes en la memoria declarativa que los niños $(t(123)=-11.08, p<0.001$, Niños: $M=4.72$, $D E=0.56$. Adolescentes: $M=6.81, D E=1.379)$.

No se encontraron diferencias significativas en la intensidad de uso televisivo, pero sí en la calificación de la serie, resultando que los niños son los que la califican mejor. $(t(116)=-2.53, p<0.05, \mathrm{Ni}-$ ños: $M=8.98, D E=1.9$. Adolescentes: $M=7.91$, $D E=2.65)$.

En lo referente a las variables psicomediáticas, se encontraron diferencias significativas en las escalas de motivación y de identificación parasocial. Los niños 
TABLA 3

Diferencias de medias entre los grupos para las variables en estudio (pruebas t)

\begin{tabular}{lcccccc}
\hline & \multicolumn{7}{c}{ Niños/as } & \multicolumn{2}{c}{ Adolescentes } \\
\cline { 2 - 7 } & $\mathrm{M}$ & $\mathrm{DE}$ & $\mathrm{M}$ & $\mathrm{DE}$ & $t$ & $\mathrm{gl}$ \\
\hline Complejidad del esquema 1 & 0.34 & 0.81 & 1.84 & 1.35 & $-7.55^{* * *}$ & 123 \\
Complejidad del esquema 2 & 1.72 & 0.79 & 1.57 & 0.94 & $0.98(\mathrm{~ns})$. & 123 \\
Memoria & 4.72 & 0.56 & 6.81 & 1.38 & $-11.02^{* * *}$ & 123 \\
Complejidad de la Narración & 95.37 & 143.06 & 92.65 & 62.61 & $0.14(\mathrm{~ns})$. & 123 \\
Intensidad de uso de la TV & 36.89 & 18.71 & 35.37 & 14.75 & $0.50(\mathrm{~ns})$. & 122 \\
Valoración de la serie & 8.98 & 1.90 & 7.91 & 2.65 & $2.53^{*}$ & 116 \\
Motivación & 17.48 & 4.86 & 15.4 & 4.44 & $2.27^{*}$ & 102 \\
Identificación Parasocial & 19.9 & 5.6 & 17.07 & 5.06 & $2.83^{* *}$ & 113 \\
Implicación & 29.65 & 7.4 & 28.18 & 5.86 & 1.11 (ns.) & 100 \\
\hline
\end{tabular}

$* p<0.05 ; * * p<0.01 ; * * p<0.001$.

Fuente: elaboración propia.

tienen mayores puntajes en la motivación hacia el visionado televisivo que los adolescentes $(t(102)=2.27$, $p<0.05$, Niños: $M=17.48, D E=4.86$. Adolescentes: $M=15.4, D E=4.44)$. De igual manera, los niños presentan mayores puntajes en identificación parasocial $(t(113)=2.83, p<0.001$, Niños: $M=19.9$, $D E=5.6$. Adolescentes: $M=17.07, D E=5.06)$.

\section{Predictores de la complejidad del esquema: el impacto de las variables cognoscitivas y psicomediáticas}

Se procedió a realizar un modelo de regresión para el personaje 1 y para el personaje 2 en niños y adolescentes, respectivamente. Se recurrió al método stepwise, considerando como variables predictoras la intensidad de uso televisivo, la valoración de la serie, las variables cognoscitivas (memoria, complejidad de la narración) y psicomediáticas (motivación implicación e identificación parasocial (Tabla 4).

En el caso de los niños, para el personaje 1 no resultó significativo el modelo. Para el personaje 2, la motivación hacia el visionado televisivo resultó significativa $\left(R^{2}=0.13, F_{(1,40)}=5.93, p<0.05\right)$, lo que indica que una alta motivación se asocia con una mayor complejidad del esquema de este personaje $(\beta=0.36, p=<0.05)$. La intensidad de uso, la valoración de la serie así como las variables cognoscitivas se excluyeron del modelo.
En lo que respecta a los adolescentes, es una de las variables cognoscitivas la que se presentan como predictoras en el modelo. La intensidad de uso y las variables psicomediáticas fueron excluidas. Específicamente, fue la complejidad narrativa la que resultó significativa para ambos personajes (Personaje 1: $R^{2}=0.14, F_{1,38}=6, p<0.05$. Personaje $\left.2: R^{2}=0.16, F_{2,38}=7.27, p<0.05\right)$, de modo que ha mayor complejidad en la estructuración de la narración sobre la serie, mayor la complejidad del esquema de este personaje $(\beta=0.37, p=<0.05$, para el personaje 1 y para el personaje $2(\beta=0.4$, $p=<0.05)$.

\section{Discusión}

Las correlaciones bivariadas sugieren un comportamiento diferencial de las variables en estudio por grupo de edad. En el grupo de adolescentes las variables cognoscitivas, específicamente la complejidad de la narración, está asociada positivamente. Caso contrario sucede en el grupo de niños, donde ninguna de estas variables se relacionó con la organización del esquema. En el caso de las variables psicomediáticas, ligadas a aspectos emocionales e interactivos de la recepción televisiva, los niños presentan más asociaciones con dichas variables en comparación con los adolescentes. En esta dirección, se encontró que la complejidad del esquema se asocia con las variables cognoscitivas, en el caso 
TABLA 4

Regresión lineal de la complejidad del esquema considerando la intendidad de uso televisivo, la valoración de la serie, las variables cognoscitivas y psicomediáticas en estudio

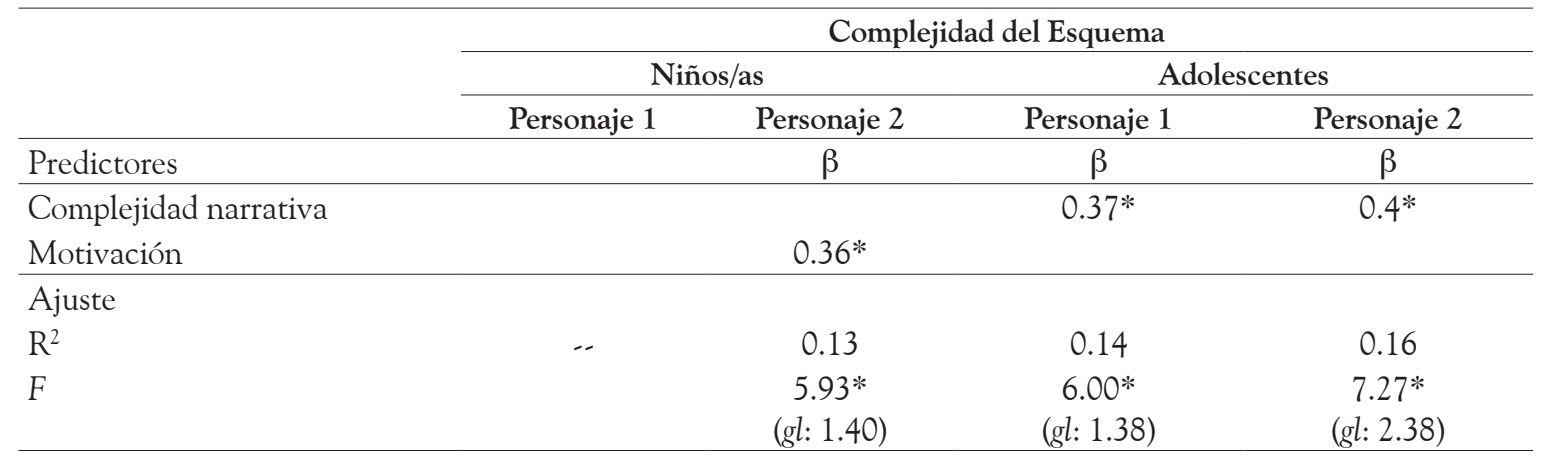

${ }^{*} p<0.05$.

Fuente: elaboración propia.

de los adolescentes, y con las variables psicomediáticas, en el caso de los niños. Este resultado sugiere, nuevamente, la apropiación diferencial del mensaje dependiendo del grupo de edad, de modo que en los niños la valoración socioemocional y la motivación va a ser la predominante, mientras que en el caso de los adolescentes, son las especificidades del procesamiento cognoscitivo individual del mensaje, las que estarían definiendo la apropiación de la serie. Será necesario, sin embargo, mayor investigación al respecto.

Las diferencias de media apuntan a que la complejidad de los esquemas es mayor en adolescentes que en los niños, presentando los adolescentes también un mayor empleo de la memoria declarativa. Así mismo, los niños presentan una valoración más positiva de la serie, así como una mayor motivación e identificación con los caracteres mediáticos.

En cuanto a los modelos de regresión, se encontró que las principales variables predictoras, la intensidad de uso televisivo y la valoración de la serie, no resultaron tener una asociación significativa con la complejidad del esquema. En el caso de los niños, fue la motivación hacia el visionado televisivo, la variable que se asoció significativamente con la complejidad del esquema para el personaje 2. Aquí resulta relevante considerar el papel de los encuadres mediáticos asociados a la caracterización de los personajes, como un posible elemento que define la estructuración del esquema (Scheufele, 1999). En la serie infantil en estudio, el personaje 2 es Cody, uno de los hermanos gemelos, que es caracterizado como una persona ingenua, inteligente, estudiosa, cortés, más bien tímida. Es posible que un tipo social con estas características sea un aspecto que genere identificación con el personaje para aquellos niños con mayor motivación hacia el uso de la televisión, expresándose en una mayor implicación cognoscitiva al caracterizar al personaje.

En lo que se refiere a los adolescentes, y como ya se había señalado al describir las correlaciones, son las variables cognoscitivas las que definen la complejidad del esquema. Específicamente, la complejidad de la narración, vale decir las competencias narrativas del o la joven, la que estructura la complejidad del esquema. Este resultado puede indicar una relación más distanciada y racional con respecto a los personajes y la serie, independientemente de la intensidad de visionado televisivo, la valoración de la serie o las otras variables psicomediáticas en estudio.

A partir de estos resultados, se puede indicar que la $\mathrm{H}_{1}$ encuentra correlato empírico, al identificarse que los adolescentes presentan mayor complejidad en los esquemas. Se encontraron indicios en el sentido de la hipótesis $\mathrm{H}_{2}$ que supone una mayor participación de la valoración de la serie en la complejidad de la serie, para el caso de los niños. Estos resultados requieren, sin embargo, de mayor investigación. $\mathrm{La} \mathrm{H}_{3}$ acerca del papel de la intensidad 
de uso televisivo, no encuentra soporte empírico. Se encontró evidencia que respalda las $\mathrm{H}_{4}$ y $\mathrm{H}_{5}$ al encontrarse, por un lado, una asociación positiva entre la memoria declarativa y la complejidad del esquema, en el grupo de adolescentes; y, por otro lado, una asociación positiva entre la motivación de visionado y la complejidad del esquema, en el grupo de niños.

Estos resultados indican la existencia de una lógica de desarrollo en la organización de los esquemas de los personajes mediáticos que van de la mano con el desarrollo de las competencias mediáticas y de las cogniciones sociales. Autores como van Evra (1998), Groeben y Hurrelman (2002) o Subrahmanyam y Greenfield (2008) han sistematizado los procesos cognoscitivos y el desarrollo asociado a la atención, comprensión, retención y recuperación de la información asociada a la televisión. El procesamiento y comprensión del mensaje mediático estaría ligado al reconocimiento de los formatos televisivos, la diferenciación entre realidad y ficción, la comparación entre esquemas de rol o de personas en la "vida real" y en el contexto televisivo, así como la comprensión de la estructura narrativa y la formación de estereotipos asociados a la categorización social de los personajes. Todos estos aspectos van a variar según la edad de las personas, resultando que la tendencia es hacia una comprensión más diferenciada de los contenidos televisivos, una distinción más clara entre realidad y ficción y una formación mucho más compleja y variada del contenido y organización de los esquemas cognitivos de personas, tal como se pudo evidenciar en los resultados de este estudio (Collins, 1981; Wright et al., 1997).

Al respecto, el presente estudio ha encontrado, de forma consistente con estudios previos, un mayor involucramiento de los niños hacia la serie en comparación con los adolescentes, y con ello una mayor capacidad de recuperación del contenido mediático, la cual está asociada al hecho de que los niños perciben lo que acontece en las series de forma más realista que los adolescentes. Esto puede explicar la mayor vinculación emocional con la serie, expresado en la asociación encon- trada entre la valoración de la serie, los factores motivacionales y la complejidad de los esquemas de personajes.

A la par de estos elementos propios del desarrollo de las llamadas competencias mediáticas, se encuentran las particularidades del desarrollo cognoscitivo, en los que igualmente se puede identificar una tendencia hacia procesos atencionales más prolongados y estructurados, una mayor capacidad de procesamiento memorístico, una comprensión y organización narrativa más compleja, así como una percepción social, que a partir de los mecanismos propios del desarrollo de una Teoría de la Mente, favorece una comprensión y evaluación de los personajes televisivos (Flavell, 2004; Goswami, 2002). Estos cambios se expresan en los resultados del presente estudio, en una mayor participación de la memoria declarativa en la organización de los esquemas.

Ahora bien, para futuras investigaciones puede resultar relevante estudiar estos resultados en términos del efecto de presencia (Lee, 2004), es decir, la tendencia a considerar el contenido mediático como algo que acontece en la actualidad y de la que se forma parte. Al respecto, podría hipotetizarse que el efecto de presencia es mayor en los niños que en los adolescentes.

Resulta igualmente relevante prestar atención al desarrollo emocional implicado en la recepción y apropiación del material mediático, el cual precisamente puede estar ligado al efecto de presencia. Estas diferencias por edad son las que podría explicar ese aparente distanciamiento del material que se contempla en los adolescentes.

Una limitación del estudio fue la imposibilidad de utilizar una misma serie para comparar entre los dos grupos de edad, limitación que, como se indicó con anterioridad, se debió a que ambos grupos consumen contenidos televisivos diferentes, además de que las series vistas por los adolescentes no eran aptas para niños, y las series de televisión vistas por los niños resultaban poco atractivas para los adolescentes. Será una tarea para futuras investigaciones, exponer a ambos grupos a una misma serie, y así tener mayor certeza de las diferencias encontradas en esta investigación. 


\section{Referencias}

Busselle, R. W. (2001). The role of exemplar accessibility in social reality judgments. Media Psychology, 3(1), 43-67.

Calvert, S. \& Huston, A. (1987). Television and children's gender schemata. New Directions for Child and Adolescent Development, 38, 75-88.

Collins, A. (1981). Schemata for understanding television. New Directions for Child and Adolescent Development, 13, 31-45.

Evra, J., van (1998). Television and child development. Mahwah, NJ: Laurence Erlbaum Associates.

Fiske, S. \& Taylor, S. (2008). Social cognition: From brain to culture. New York: McGraw-Hill.

Flavell, J. (2004). Theory-of-Mind development: Retrospect and prospect. Merrill-Palmer Quarterly, 50(3), 274-290.

Ford, T. (1997). Effects of stereotypical perception portrayals of Afro-Americans on person perception. Social Psychology Quarterly, 60(3), 266-275.

Goswami, U. (2002). Blackwell handbook of childhood cognitive development. Oxford, UK: Blackwell Publishing Ltd.

Groeben, N. \& Hurrelman, B. (2002). Medienkompetenz: Voraussetzungen, Dimensionen, Funktionen. [Competencia mediática: presupuestos, dimensiones, funciones]. Weinheim, München: Juventa.

Harwood, J. (1999). Age identification, social identity gratifications, and television viewing. Journal of Broadcasting \& Electronic Media, 43(1), 123-136.

Krippendorf, K. \& Rock, M. (2008). The content analysis reader. Thousand Oaks, CA: Sage Publications.

Lee, K. M. (2004). Presence, explicated. Communication Theory, 14(1), 27-50.

Mastro, D. (2003). A social identity approach to understanding the impact of television messages. Communication Monographs, 70(2), 98-113.

Mastro, D. \& Kopacz, M. (2006). Media representations of race, prototypicality, and policy reasoning: An application of self-categorization theory. Journal of Broadcasting \& Electronic Media, 50(2), 305-322.

Pérez, R. (2003a). Televisión, juventud y cambio cultural: recepción de series de televisión y la construcción de representaciones acerca del futuro en jóvenes costarricenses y alemanes. Actualidades en Psicología, 18, 17-48.

Pérez, R. (2003b). Psicología social de la comunicación de masas: introducción a las teorías psicosociales de la comunicación de masas. San José: Universidad de Costa Rica, Sistema Editorial de Difusión Científica de la Investigación.

Rubin, A. (2002). Uses and gratifications perspective of media effect. En J. Bryant \& D. Zillmann (Eds.), Media effects: Advances in theory and research (pp. 525-548). Mahwah, NJ: Lawrence Erlbaum Associates.

Rubin, A. \& Perse, E. (1987). Audience activity and soap opera involvement: A uses and effects investigation. Human Communication Research, 14(2), 246-268.

Scheufele, D. (1999). Framing as a theory of media effects. Journal of Communication, 49(1), 103-122.

Shanahan, J. \& Morgan, M. (2003). Television and its viewers. Cultivation theory and research. Cambridge: Cambridge University Press.

Shrum, L. J. (2006). Perception. En J. Bryant \& P. Vorderer (Eds.), Psychology of Entertainment (pp. 5570). Mahwah, NJ: Lawrence Erlbaum Associates.

Subrahmanyam, K. \& Greenfield, P. (2008). Media Symbol Systems and Cognitive Processes. En S. 1. Calvert \& B. J. Wilson (Eds.), The handbook of children, media, and development (pp. 166-186). Oxford, UK: Blackwell Publishing Ltd.

Ward, M. \& Rivadeneyra, R. (1999). Contributions of entertainment television to adolescents' sexual attitudes and expectations: The role of viewing amount versus viewer involvement. The Journal of Sex Research, 36(3), 237-249.

Wicks, R. H. (2006). Media Information Processing. En J. Bryant \& P. Vorderer (Eds.), Psychology of Entertainment (pp. 85-102). Mahwah, NJ: Lawrence Erlbaum Associates.

Willis, G. (2004). Cognitive interviewing: A tool for improving questionnaire design. Thousend Oaks, CA: Sage Publications.

Wright, J., Huston, A., Fitch, M., Wroblewski, R. \& Piemyat, R. (1997). Effects of documentary and fictional television formats on children's acquisition of schemata for unfamiliar occupations. Journal of Applied Developmental Psychology, 18(4), 563-585. 
Wright, J., Huston, A., Truglio, R., Flitch, M., Smith, E. \& Piemyat, S. (1995). Occupational portrays on television: Children role schemata, career aspirations and perception of reality. Child Development, 66(6), 1706-1718.
Wroblewski, R. \& Huston, A. (1987). Televised occupational stereotypes and their effects on early adolescents: Are they changing? The Journal of Early Adolescence, 7(3), 283-297. 